\title{
Repeated Transcranial Direct Current Stimulation Prevents Abnormal Behaviors Associated with Abstinence from Chronic Nicotine Consumption
}

\author{
Solène Pedron', Julie Monnin ${ }^{1,2}$, Emmanuel Haffen ${ }^{1,2}$, Daniel Sechter' and Vincent Van Waes*,' \\ 'EA 481 Laboratory of Integrative and Clinical Neuroscience, University of Franche-Comté/SFR FED 4234, Besançon, France; \\ 2INSERM CIC-IT 808 Clinical Investigation Centre for Innovative Technology, University Hospital of Besançon, Besançon, France
}

\begin{abstract}
Successful available treatments to quit smoking remain scarce. Recently, the potential of transcranial direct current stimulation (tDCS) as a tool to reduce craving for nicotine has gained interest. However, there is no documented animal model to assess the neurobiological mechanisms of tDCS on addiction-related behaviors. To address this topic, we have developed a model of repeated tDCS in mice and used it to validate its effectiveness in relieving nicotine addiction. Anodal repeated tDCS was applied over the frontal cortex of Swiss female mice. The stimulation electrode (anode) was fixed directly onto the cranium, and the reference electrode was placed onto the ventral thorax. $\mathrm{A}$ $2 \times 20 \mathrm{~min} /$ day stimulation paradigm for five consecutive days was used $(0.2 \mathrm{~mA})$. In the first study, we screened for behaviors altered by the stimulation. Second, we tested whether tDCS could alleviate abnormal behaviors associated with abstinence from nicotine consumption. In naive animals, repeated tDCS had antidepressant-like properties 3 weeks after the last stimulation, improved working memory, and decreased conditioned place preference for nicotine without affecting locomotor activity and anxiety-related behavior. Importantly, abnormal behaviors associated with chronic nicotine exposure (ie, depression-like behavior, increase in nicotine-induced place preference) were normalized by repeated tDCS. Our data show for the first time in an animal model that repeated tDCS is a promising, non-expensive clinical tool that could be used to reduce smoking craving and facilitate smoking cessation. Our animal model will be useful to investigate the mechanisms underlying the effects of tDCS on addiction and other psychiatric disorders.
\end{abstract}

Neuropsychopharmacology (2014) 39, 98I-988; doi: I0. I038/npp.20 I3.298; published online 20 November 2013

Keywords: neuromodulation; nicotine withdrawal; depression; conditioned place preference; memory; addiction

\section{INTRODUCTION}

Transcranial direct current stimulation (tDCS) is a neuromodulatory technique that consists in stimulating the cerebral cortex with a weak constant electric current in a non-invasive and painless manner. Since a decade ago, tDCS has been used experimentally to treat several psychiatric disorders, including depressive symptoms (Brunoni et al, 2012; Nitsche et al, 2009). The mechanisms underlying its effects are not well understood, but early studies in animals using current directly applied to the cortex suggest that anodal stimulation causes a depolarization of the resting membrane potential and increases the firing rates of cortical neurons in the tissue under the electrode (Bindman et al, 1964; Purpura and McMurtry, 1965; Stagg and Nitsche, 2011).

Recently, the use of tDCS-induced modulation of cortical excitability has gained interest in the scientific community

\footnotetext{
*Correspondence: Dr $\vee$ Van Waes, Laboratory of Integrative and Clinical Neuroscience, University of Franche-Comté-UFR Sciences et Techniques, 2 Place Leclerc, 25030 Besancon, Cedex, France,

Tel: (+33)38|665726, Fax: (+33)381665746,

E-mail: vincent.van_waes@univ-fcomte.fr

Received 29 July 2013; revised 2 October 2013; accepted 16 October 2013; accepted article preview online 24 October 2013
}

as a means to decrease maladaptive behaviors in drugdependent patients (Feil and Zangen, 2010). Two preliminary clinical studies carried out in chronic smokers support/ exemplify tDCS efficacy in relieving addiction-related behaviors (Boggio et al, 2009; Fregni et al, 2008). In these studies, the dorsolateral prefrontal cortex (DLPFC) was targeted because smoking craving processing has been associated with enhanced activity of this area (Brody et al, 2002; Due et al, 2002; McBride et al, 2006; Wilson et al, 2004). In the first clinical trial, Fregni et al (2008) showed that a single-tDCS session over the DLPFC reduced cueinduced smoking craving in tobacco users. Participants received three different types of tDCS: sham tDCS, anodal tDCS of the left DLPFC, and anodal tDCS of the right DLPFC (a single session of $2 \mathrm{~mA}$ for $20 \mathrm{~min}$ ). Before and right after the electrical stimulation, they completed a visual analog scale (VAS) to evaluate mood and a nicotine-based VAS to measure craving levels. The authors found that stimulation of both left and right DLPFC with active, but not sham, tDCS reduced general and smoking cue-induced nicotine craving with no other significant mood changes associated with the tDCS treatment. In the second study (Boggio et al, 2009), chronic smokers were randomized to receive either sham tDCS or active anodal tDCS of the left DLPFC for five 
consecutive days ( $2 \mathrm{~mA}, 1$ session of $20 \mathrm{~min}$ per day). The results indicated a cumulative effect of tDCS such that the magnitude of tDCS on reducing cue-induced craving increased after each session. Nonetheless, the duration of this phenomenon was not evaluated in this work.

These preliminary findings are very encouraging because current available treatment options for smoking cessation remain limited and/or are associated with poor long-term success rates (O'Brien, 2008). Several approaches, such as nicotine replacement therapy (patch, chewing gum, electronic cigarette), drugs (eg, brupopion, varenicline), and psychotherapy (behavioral and motivational interventions) are used for the purpose of decreasing smoking craving (see Stead and Lancaster, 2012); however, their relative poor efficacy and potential drug side effects limit the use of these methods. In this context, electrical stimulation of specific brain regions appears as innovative and attractive technique worth of further investigation to reduce smoking craving (Fraser and Rosen, 2012).

In spite of these promising results, a suitable experimental animal model with translational value is presently lacking in order to investigate the mechanisms underlying tDCS effects on addiction-related behavior. The aim of the present work is to characterize an animal model that can be used to study the neurobiological mechanisms associated with the efficacy of the tDCS treatment. The first step toward this goal is to demonstrate that protocols of tDCS in laboratory animal can result in positive/negative outcomes on a variety of behavioral measures that are clinically relevant. On the basis of the work of Liebetanz et al (2006; 2009), we have developed a model of repeated tDCS in mice with an experimental paradigm similar to that used in clinical trials. In a first experiment, we screened naive animals (never exposed to nicotine) for behaviors altered by repeated tDCS (depression, anxiety, memory, and reinforcing effect of nicotine). In a second set of experiments, we tested whether tDCS could alleviate behaviors associated with abstinence from chronic nicotine consumption during adolescence (postnatal day, PND 30-43), a period of high vulnerability to nicotine exposure (Iniguez et al, 2009). Our data show for the first time in an animal model that repeated tDCS has antidepressant properties and decreases the reinforcing effect of nicotine consistent with the outcomes observed after repeated tDCS in humans.

\section{MATERIALS AND METHODS}

\section{Subjects}

Swiss female mice (Janvier, Laval, France) were housed 4-5 per cage under standard laboratory conditions (12:12-hour light/ dark cycle; lights on at 0700 hours) with food and water available ad libitum. Before the surgery, mice were allowed 1 week of acclimation, during which they were repeatedly handled. All procedures met the NIH guidelines for the care and use of laboratory animals and were approved by the University of Franche-Comté Animal Care and Use Committee.

\section{Surgery}

A tubular plastic jacket (internal diameter: $2.1 \mathrm{~mm}$ ) was surgically fixed onto the skull 1 week before the stimulation



Figure I Illustration of the tDCS device used to deliver the current stimulation. (a) The mouse is placed in a custom-made restraint box. The anode (contact area $3.5 \mathrm{~mm}^{2}$ ) is positioned over the left frontal cortex and the cathode (rubber-plate electrode, $9.5 \mathrm{~cm}^{2}$ ) onto the ventral thorax. A $2 \times 20 \mathrm{~min} /$ day constant current of $0.2 \mathrm{~mA}$ is applied transcranially using a direct current stimulator (DC-Stimulator Plus) for five consecutive days, with a linear fade in/fade out of IOs. (b) The center of the electrode is positioned over the left frontal cortex $1 \mathrm{~mm}$ anterior to the coronal fissure and I mm left of the sagittal fissure (adapted from Paxinos and Franklin, 200 I). (c) Anode: a tubular plastic jacket (internal diameter: $2.1 \mathrm{~mm}$ ) is surgically fixed onto the skull and filled with saline solution before the stimulation. The stimulation electrode is screwed into the tubular plastic jacket and dip in the saline solution. Only the saline solution is in contact with the skull.

protocol (Figure 1). Animals were anesthetized with ketamine hydrochloride/xylazine $(80 \mathrm{mg} / 12 \mathrm{mg} / \mathrm{kg}$, respectively; i.p.) and were placed in a stereotaxic apparatus. The center of the plastic jacket was positioned over the left frontal cortex $1 \mathrm{~mm}$ anterior to the coronal fissure and $1 \mathrm{~mm}$ left of the sagittal fissure (Figure $1 \mathrm{~b}$ ) and fixed with a coating of glass ionomer cement (GC Fuji I, Leuven, Belgium). After surgery, all animals were allowed to recover for 1 week before undergoing tDCS. During this period as well as during the electrical stimulations, mice were placed in individual cages. Experimental design of experiments 1 and 2 is summarized in Figures $2 \mathrm{a}$ and $3 \mathrm{a}$.

\section{Stimulation Protocol}

The jacket was filled with saline solution $(\mathrm{NaCl} 0.9 \%)$ before the stimulation to establish a contact area of $3.5 \mathrm{~mm}^{2}$ toward the skull. The stimulation electrode (anode, Figure 1c, DIXI Medical, Besançon, France) was screwed into the tubular plastic jacket. A larger conventional rubberplate electrode (cathode, $9.5 \mathrm{~cm}^{2}$; Physiomed Elektromedizin AG, Schnaittach, Germany) served as the counterelectrode and was placed onto the ventral thorax (adapted from Liebetanz et al, 2009, Figure 1a). This setting prevented the bypassing of currents (shunting effect) that would occur in the case of two juxtaposed encephalic electrodes in mice. An anodal $2 \times 20 \mathrm{~min} /$ day constant current of $0.2 \mathrm{~mA}$ was applied transcranially over the frontal cortex using a DC-Stimulator Plus (NeuroConn, Ilnemau, Germany) for five consecutive days, with a linear fade in/ fade out of 10s (Ferrucci et al, 2009; Rigonatti et al, 2008). Animals were awake and restrained during the tDCS (Figure 1a) to prevent possible interactions between tDCS effects and anesthetic drugs. Control animals were subjected 
a

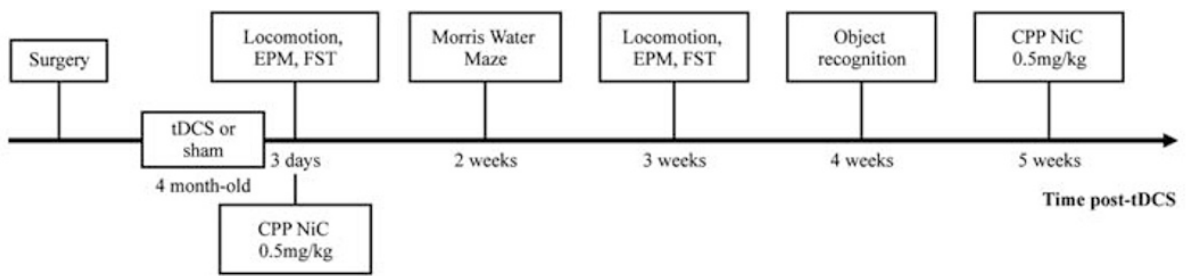

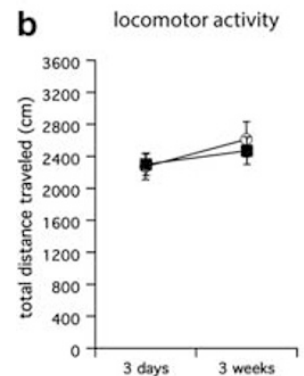

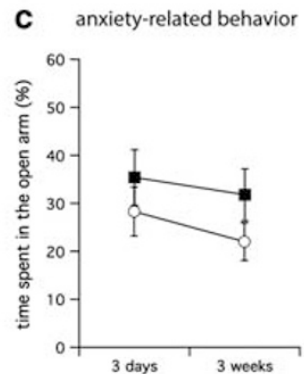

e addiction-related behavior

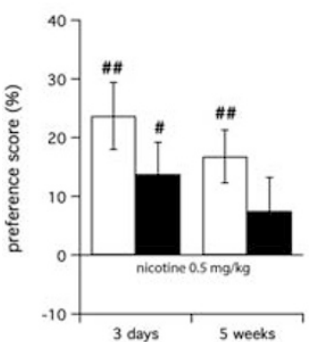

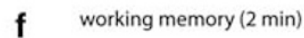

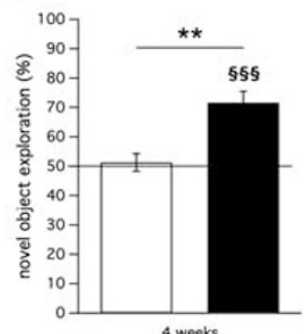

d depression-related behaviors
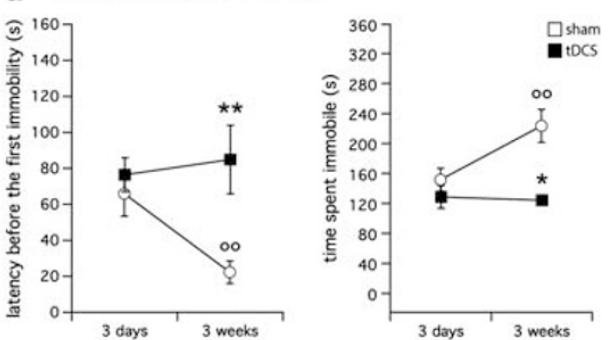

g long-term spatial memory
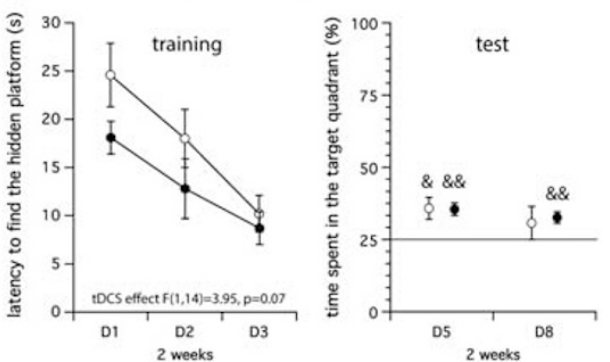

Figure 2 Experiment I. (a) Experimental design. Four-month-old Swiss female mice never exposed to nicotine were subjected to repeated anodal tDCS for five consecutive days $(2 \times 20 \mathrm{~min} /$ day constant current, $0.2 \mathrm{~mA})$. Behavioral effects of tDCS were screened from 3 days to 5 weeks following the last electrical stimulation. The same animals were used in all behavioral tests (sham $N=8, t D C S N=8$ ) except in the CPP test at 3 days for which another batch of mice was used (sham $N=10$, tDCS $N=10$ ). EPM: elevated plus maze, FST: forced swim test, CPP: conditioned place preference, NiC: nicotine. Effect of repeated anodal tDCS on (b) locomotor activity, (c) anxiety-related behavior, (d) depression-related behaviors, and (e) addiction-related behavior 3 days and 3-5 weeks following the last electrical stimulation. (f) Effect of repeated anodal tDCS on working memory 4 weeks after the last stimulation and (g) effect of repeated anodal tDCS on long-term spatial memory (training, left; test, right) 2 weeks after the last stimulation. * $p<0.05$ and $* * p<0.0$ I vs sham, ${ }^{\circ 0} p<0.013$ days vs 3 weeks, ${ }^{\#} p<0.05$ and ${ }^{\# \#} p<0.01$ vs $0 \%,{ }^{\$ \$ \$} p<0.001$ vs $50 \%,{ }^{\&_{p}}<0.05$ and ${ }^{\& \&} p<0.01$ vs $25 \%$.

to the same procedure (surgeries, restraint box, electrode montage), but current was not delivered.

\section{Nicotine Treatment during Adolescence}

Adolescent mice (PND 30-43) were treated twice a day for 14 consecutive days with nicotine $(1 \mathrm{mg} / \mathrm{kg}$ i.p. in $0.2 \mathrm{ml}$, time between injections: $6 \mathrm{~h}$; $(-)$-nicotine hydrogen tartrate salt, SIGMA, France; Figure 3a). Control mice were injected with $\mathrm{NaCl} 0.9 \%$ following the same schedule.

\section{Behavioral Tests}

Body weight, food, and water consumption. The weight of mice and food/fluid intake during the 24 -h period were monitored throughout the experiments.

Locomotor activity. A system that automatically analyzes locomotor activity of mice in a cage similar to their home cage (Activmeter, Bioseb, France) was used to record the total distance traveled (in $\mathrm{cm}$ ) by the mice during $16 \mathrm{~min}$. This system consists of a transparent plastic cage $(17 \times$ $21 \times 14 \mathrm{~cm}$ ) and uses cage vibrations to measure locomotion.
Elevated plus maze. Anxiety-related behavior was examined in the elevated plus maze (EPM) test (Walf and Frye, 2007). The arms (two open without walls and two enclosed by $45 \mathrm{~cm}$ high walls) were $30 \mathrm{~cm}$ long, $6 \mathrm{~cm}$ wide, and raised off the floor by $50 \mathrm{~cm}$. Each animal was placed in the center square of the apparatus facing a closed arm and was allowed to move freely for $5 \mathrm{~min}$. The test was recorded with a video camera and analyzed using the Ethovision system (videotracking, Noldus, Paris, France). The parameter used to assess anxiety-related behavior was the percentage of time spent in the open arms during the $5 \mathrm{~min}$.

Forced swim test. We adapted the forced swim test (FST) method from Porsolt et al (1977). Each mouse was placed into a beaker (height $26 \mathrm{~cm}$, diameter $18 \mathrm{~cm}$ ) containing water at a temperature of $32 \pm 2{ }^{\circ} \mathrm{C}$ and a depth of $17 \mathrm{~cm}$ so that the mouse could neither escape nor touch the bottom. Each test lasted $6 \mathrm{~min}$ and was video recorded for subsequent scoring by a blind observer of the latency before the first episode of immobility and the total time spent immobile. Mice were considered immobile when they ceased struggling and remained floating motionless in the water for at least $2 \mathrm{~s}$. 


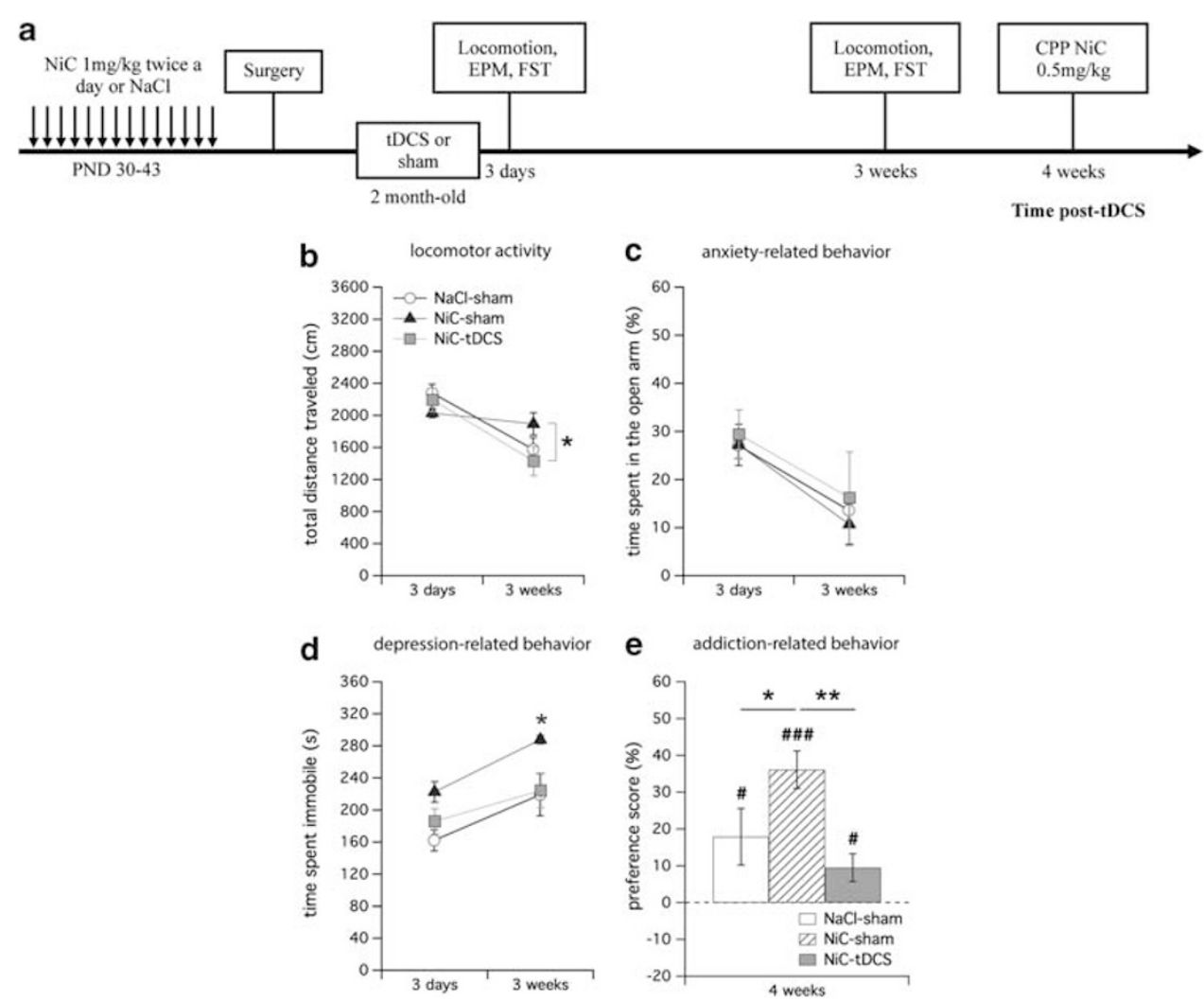

Figure 3 Experiment 2. (a) Experimental design. Adolescent mice were treated twice a day for 14 consecutive days with nicotine (I mg/kg i.p.) postnatal day (PND) 30-43. Control mice were injected with $\mathrm{NaCl} 0.9 \%$ following the same schedule. Nicotine and NaCl-treated animals were submitted to repeated anodal tDCS or sham stimulations for five days (PND 60-64). Animals were then tested for locomotor activity, anxiety- and depression-related behaviors, and for their sensitivity to the rewarding effects of nicotine. CPP, conditioned place preference; EPM, elevated plus maze; FST, forced swim test; $\mathrm{NiC}$, nicotine. $\mathrm{NaCl}$-sham $(\mathrm{N}=10), \mathrm{NiC}$-sham $(\mathrm{N}=9), \mathrm{NiC}$-tDCS $(\mathrm{N}=10)$. (b) Effect of nicotine exposure during adolescence and subsequent repeated anodal tDCS on locomotor activity, (c) anxiety-related behavior, (d) depression-related behaviors, and (e) addiction-related behavior 3 days and 3-4 weeks following the last electrical stimulation. ${ }^{*} p<0.05$ and ${ }^{* *} p<0.0 I$ vs NaCl-sham or as indicated, ${ }^{\#} p<0.05$ and ${ }^{\# \# \# ~} p<0.00$ I vs $0 \%$.

Novel object recognition task. Two objects (figurines) with different forms and colors were used for the experiments. There were two copies of each object. A preliminary test was carried out to verify there was no preexisting preference for any of the figurines. Mice were habituated to the empty test arena (diameter: $47 \mathrm{~cm}$ ) $10 \mathrm{~min}$ per day for four consecutive days before the object recognition test. During the exposure phase, two identical copies of the sample object were placed in the arena, and mice were allowed to explore the objects for $10 \mathrm{~min}$. After an intertrial interval of $2 \mathrm{~min}$ in the home cage, mice were placed again in the test arena for 5 min (test phase). During this phase, the arena contained one object used in the exposure phase and one novel object. The arena and objects were wiped down with $70 \%$ ethanol between trials to minimize olfactory cues. Novel object exploration during the test phase was determined as the percentage of time spent with the nose not more than $1 \mathrm{~cm}$ away from the novel object divided by the total time spent to explore the two objects. The test was recorded with a video camera and analyzed using the Ethovision system.

Morris water maze. A circular pool (diameter: $130 \mathrm{~cm}$, height: $30 \mathrm{~cm}$ ) was filled to a depth of $10 \mathrm{~cm}$ with water $\left(32 \pm 2{ }^{\circ} \mathrm{C}\right)$ and placed in a room with visual cues. Over three consecutive days, mice were given 12 training trials per day. A clear platform (diameter: $9 \mathrm{~cm}$ ) was placed at the midpoint of one quadrant, submerged $0.5 \mathrm{~cm}$ below the water surface, and fixed in the same place throughout the training trials. The point of entry of the mouse into the pool was randomized. When a mouse located the platform, it was allowed to remain on it for $20 \mathrm{~s}$. If the mouse could not locate the platform within $60 \mathrm{~s}$, it was gently navigated to the platform and remained on it for $20 \mathrm{~s}$. The parameter evaluated was the latency to find the hidden platform in seconds. A test trial was then carried out 2 and 5 days after the last training trial. During the test trial, the platform was removed, and mice were allowed to swim freely for $60 \mathrm{~s}$. The percentage of time spent in the target quadrant (containing the hidden platform during the training trials) was recorded and compared with $25 \%$. The test was monitored using the Ethovision system.

Nicotine-induced conditioned place preference. The conditioned place preference apparatus consists of two main compartments linked by a corridor displaying each different features: visual (wall patterns) and tactile (floor texture). On day 1 (pre-conditioning), mice were placed in the corridor and allowed free access to the compartments for $10 \mathrm{~min}$. The time spent in each compartment was recorded using the 
Ethovision system. On days 2-4 (conditioning phase) mice received injections of nicotine $(0.5 \mathrm{mg} / \mathrm{kg}$, i.p.) or vehicle and were immediately confined into one of the two conditioning compartments for $15 \mathrm{~min}$ (drug pairing was biased to the least preferred chamber). On day 5 (post-conditioning), mice were once again allowed free access to the compartments for $10 \mathrm{~min}$. Percentage of time spent in the drug-paired compartment was calculated during the pre-conditioning and the post-conditioning phases as follows: drug-paired compartment (s)/(drug-paired compartment (s) $+\mathrm{NaCl}$ paired compartment $(\mathrm{s})) \times 100$. The preference score was determined as the difference between the percentages of time spent in the drug-paired compartment during the postconditioning and pre-conditioning sessions.

\section{Statistical Analysis}

The results were expressed as mean \pm SEM. Significance was set at $p \leqslant 0.05$. Repeated analysis of variance (ANOVA) was used to analyze body weight, food/fluid consumption, locomotor activity, anxiety-, and depression-related behaviors with group as between-subject variable (experiment 1: sham, tDCS; experiment 2: NaCl-sham, NiC-sham, NictDCS) and time as within-subject variable (3 days, 3 weeks). Repeated ANOVA was also used to analyze long-term spatial memory in experiment 1 with tDCS as betweensubject variable (sham, tDCS) and time as within-subject variable (D1, D2, D3; D5, D8). Student's $t$-test (experiment 1) or one-way ANOVA (experiment 2) was used to analyze addiction-related behavior at 3 days, 4 , or 5 weeks. Newman-Keuls post hoc tests were used to describe differences between individual groups. Student's $t$-tests were also used to compare the means of each group with a standard value (ie, $0 \%$ for the conditioned place preference, $50 \%$ for the novel object recognition task, and $25 \%$ for the test trial in the Morris water maze).

\section{RESULTS}

\section{Experiment 1}

Body weight, locomotor activity, and anxiety-related behavior. tDCS had no impact on body weight or on food and fluid consumption 3 days and 3 weeks after the last electrical stimulation (data not shown). Neither locomotor activity (total distance traveled during $16 \mathrm{~min}$, Figure 2b), nor anxiety-related behavior (percentage of time spent in the open arm of the EPM during 5 min, Figure 2c) was affected by tDCS. Although tDCS caused a slight drop in anxiety-related behavior (Figure 2c), this effect was not significant (ANOVA tDCS effect: $\mathrm{F}(1,14)=1.93, p=0.19$; time effect: $\mathrm{F}(1,14)=1.57, p=0.23$; tDCS $\mathrm{x}$ time interaction: $\mathrm{F}(1,14)=0.11, p=0.74)$.

Depression-related behaviors. In the FST, tDCS differently affected the latency before the first immobility (Figure 2d, left) and the immobility time (Figure 2d, right) depending on the time post stimulation (ANOVA tDCS $\mathrm{x}$ time interaction: $\mathrm{F}(1,14)=7.67, p<0.05$ and $\mathrm{F}(1,14)=7.14$, $p<0.05$, respectively). Post hoc Newman-Keuls analysis revealed that, relative to sham controls, tDCS significantly increased the latency before the first immobility (Figure 2d, left, ${ }^{* *} p<0.01$ ) and decreased immobility time (Figure $2 \mathrm{~d}$, right, $\left.{ }^{*} p<0.05\right) 3$ weeks after the last stimulation, but not after 3 days (all $p>0.05$ ). The sham group was affected by the repetition of the test as evidenced by a significant decrease in the latency before the first immobility and increase in the time spent immobile between 3 days and 3 weeks $\left({ }^{\circ} p<0.01\right.$, Figure $\left.2 \mathrm{~d}\right)$. A complementary experiment with a different batch of mice tested only 3 weeks after the last stimulation confirmed the antidepressive-like property of tDCS in the FST (data not shown).

Addiction-related behavior. Nicotine $(0.5 \mathrm{mg} / \mathrm{kg})$ induced a significant place preference in the sham group 3 days $(p<0.01 v s 0 \%$, Figure $2 \mathrm{e})$ and 5 weeks $(p<0.01$ vs $0 \%)$ following sham stimulations. Animals submitted to repeated anodal tDCS also displayed a place preference for nicotine 3 days after the last stimulation $(p<0.05 v s 0 \%)$. However, 3 weeks after the last stimulation, the preference score for nicotine was not significantly different from $0 \%(p=0.23)$. Student's $t$-test did not reveal a significant direct difference between sham and tDCS groups.

Working memory. The percentage of exploration of the novel object during the test phase was not significantly different from $50 \%$ in the sham group, indicating that animals did not differentiate the novel object from the object already presented with an intertrial interval of $2 \mathrm{~min}$ (sham group: $p=0.68$ s $50 \%$, Figure $2 \mathrm{f}$ ). In contrast, mice exposed to active tDCS explored significantly more the novel object than the object already presented (tDCS group: $p<0.001$ vs $50 \%$ ).

Long-term spatial memory. There was only a trend for a beneficial global effect of tDCS on learning performances (ANOVA tDCS effect: $F(1,14)=3.95, p=0.07$, Figure $2 \mathrm{~g}$, left). This effect was reflected in the test trials in which both groups spent significantly more time (compared to $25 \%$ ) in the quadrant associated with the platform (target quadrant) than in the other quadrants at day 5 (all $p<0.05$ ) but not at day 8 when only the tDCS group performed well (sham $p=0.35$, tDCS $p<0.01$ vs $25 \%$; Figure $2 \mathrm{~g}$, right). Repeated ANOVA, however, did not reveal any significant effect of tDCS on spatial memory in the test trials (ANOVA tDCS effect: $F(1,14)=0.03, p=0.86$, time effect: $F(1,14)=2.23$, $p=0.16$, tDCS $x$ time interaction: $\mathrm{F}(1,14)=0.17, p=0.68)$.

\section{Experiment 2}

Locomotor activity and anxiety-related behavior. Locomotor activity in adults tended to be affected differently by the group depending on the time post stimulation (ANOVA group $\mathrm{x}$ time interaction: $\mathrm{F}(2,26)=3.09, \quad p=0.06$, Figure 3b). Post hoc Newman-Keuls analysis indicated that tDCS significantly reduced locomotor activity in nicotinetreated animals 3 weeks after the last stimulation (NiC-sham $v s \mathrm{NiC}$-tDCS, $p<0.05)$. The percentage of time spent in the open arm of the EPM after nicotine withdrawal was not affected by the group (ANOVA group effect: $\mathrm{F}(2,26)=0.27$, $p=0.77$, group $\times$ time interaction: $\mathrm{F}(2,26)=0.05, p=0.95$, Figure 3c). 
Depression-related behaviors. The time spent immobile in the FST during adulthood was significantly affected by the experimental conditions (ANOVA group effect: $\mathrm{F}(2,26)=4.66, p<0.05$, Figure $3 \mathrm{~d})$. Post hoc NewmanKeuls analysis revealed that nicotine exposure during adolescence increased the time spent immobile in the FST during adulthood (NaCl-sham vs NiC-sham, $p<0.05)$. Exposure to repeated tDCS normalized this parameter (NiC-sham $v s$ NiC-tDCS, $p<0.05, \mathrm{NaCl}$-sham $v s$ NiC-tDCS, $p=0.51)$. When time was taken into consideration, tDCS significantly reduced the immobility in nicotine-pretreated animal only 3 weeks after the stimulation ( $\mathrm{NaCl}$-sham vs NiC-sham, $p<0.05$ and NiC-sham $v s$ NiC-tDCS at 3 weeks, $p<0.05)$. Three weeks after the stimulation, animals exposed to nicotine during adolescence and stimulated during the withdrawal period did not differ from animals never exposed to nicotine and not stimulated (NiC-tDCS $v s$ NaCl-sham, $p=0.98$ ).

Addiction-related behavior. As in experiment 1, injections of nicotine $(0.5 \mathrm{mg} / \mathrm{kg}$, i.p) induced a place preference in the sham group never exposed to nicotine (NaCl-sham, $p<0.05 v s 0 \%$, Figure 3e). Exposure to nicotine during adolescence robustly increased the place preference induced by nicotine in adults (ANOVA group effect: $\mathrm{F}(2,26)=5.21$, $p<0.05$; Nic-sham $v s \mathrm{NaCl}$-sham, $p<0.05 ; v s 0 \%, p<0.001$ ). tDCS significantly reduced nicotine-induced place preference in nicotine-pretreated animals (NiC-sham $v s$ Nic-tDCS, $p<0.01)$. Animals exposed to nicotine during adolescence and stimulated during the withdrawal period did not differ from animals never exposed to nicotine and not stimulated (Nic-tDCS vs $0 \%, p<0.05$; NiC-tDCS vs NaCl-sham, $p=0.32$ ).

\section{DISCUSSION}

The present study is the first to show that repeated anodal tDCS over the frontal lobe induces long-lasting modulation of the behavior in mice. Notably, tDCS decreases abnormal behaviors associated with abstinence in an animal model of chronic nicotine consumption. These results provide important preclinical evidence for the use of electrical brain stimulation in promoting smoking cessation and potentially ameliorating other addiction-related behaviors in dependent patients (Boggio et al, 2009; Fregni et al, 2008).

There is a growing body of evidence to suggest that tDCS has an antidepressant effect in humans (for review see Brunoni et al, 2012; Nitsche et al, 2009), but, to the best of our knowledge, this has never been shown in an animal model. Our study demonstrates that repeated anodal tDCS per se produces significant antidepressant-like effects in mice in the most frequently used test of learned helplessness ( 3 weeks after the stimulations). This effect seems relatively specific as body weight, food/drink consumption, locomotor activity, and anxiety-related behavior were unaffected both 3 days and 3 weeks following the treatment. It is known that the 'acute' physiological effects of a single tDCS session are not limited to modulation of cortical excitability during stimulation and may outlast the stimulation period by several minutes or even hours (Bindman and Richardson, 1969; Nitsche et al, 2003; Nitsche and Paulus,
2000; Ohn et al, 2008); however, the long-lasting effects of repeated stimulation on behavior have never been reported before. The effects of such repeated sessions of anodal tDCS on the immobility in the FST were present at 3 weeks but absent 3 days after the last stimulation. Interestingly, this delayed response is reminiscent of the delayed therapeutic onset of antidepressants typically reported in humans (Lam, 2012). However, in rodents, the behavioral effects of antidepressant drugs (eg, selective serotonine reuptake inhibitors, SSRIs) are usually observed acutely after a single injection, suggesting different mechanisms of action between SSRI antidepressants and tDCS.

Repeated anodal tDCS had minimal impact on spatial learning and memory in the Morris Water Maze. Conversely, our stimulation protocol significantly improved mice performance in a working memory task. This result is in accordance with a growing number of studies, indicating an improvement of working memory by anodal tDCS (Boggio et al, 2006; Fregni et al, 2005; Ohn et al, 2008). In a recent work, Zaehle et al (2011) studied working memory function in humans several minutes after a single tDCS over the DLPFC (15 min, $1 \mathrm{~mA}$ ) in combination with neurophysiological methods. Working memory was improved, and oscillatory brain activity was affected, as evidenced by amplified oscillatory power in the theta and alpha bands after the stimulation. Our study assessed for the first time the impact of repeated anodal tDCS (2 sessions per days, 5 consecutive days) and demonstrates that this protocol has long-lasting beneficial consequences in working memory of mice for at least 4 weeks. Longer time points remain to be tested to determine more precisely the duration of this effect.

Antidepressants can be used to facilitate smoking cessation (Hughes et al, 2007). What about tDCS? Chronic exposure to nicotine during adolescence induces depression-related behavior in adult rats (1 month after nicotine withdrawal (Iniguez et al, 2009)). Interestingly, Iniguez et al (2009) demonstrated that the altered behaviors observed after nicotine withdrawal can be prevented in rats by either subsequent re-exposure to nicotine or antidepressant treatment (fluoxetine or bupropion; $10 \mathrm{mg} / \mathrm{kg}$ ). Similarly, our results show that mice exposed to nicotine during adolescence display increased immobility time in the FST after nicotine withdrawal. In agreement with the previous work, this alteration was normalized by our repeated anodal tDCS protocol. Our study also shows that exposure to nicotine during adolescence dramatically increases nicotine-induced place preference $(0.5 \mathrm{mg} / \mathrm{kg})$ in adult mice, an effect that was completely abolished by the electrical stimulations.

In the brain, nicotine binds to nicotinic cholinergic receptors, which are ligand-gated ion channels that normally bind acetylcholine. Stimulation of nicotinic cholinergic receptors is known to evoke dopamine release in the cortical and subcortical dopaminergic systems, which are critical for the reinforcing effects of nicotine. This effect can be direct via the activation of dopaminergic neurons or indirect via the stimulation of other neurotransmitters' release including glutamate. Animal experiments have shown that descending pathways from the frontal cortex modulate the release of dopamine in subcortical areas such as the striatum (Karreman and Moghaddam, 1996; Taber 
and Fibiger, 1993, 1995). There is evidence that this occurs both directly via glutamatergic corticostriatal projections (Taber and Fibiger, 1995) and indirectly through an effect on mesostriatal dopamine neurons in the midbrain (Karreman and Moghaddam, 1996). As a possible mechanism of action, we hypothesize that anodal tDCS could promote a direct or indirect modulation of subcortical dopamine release involved in nicotine addiction. However, to definitively conclude that tDCS impacts nicotine consumption, it will be necessary to substantiate our findings in an intravenous self-administration paradigm.

Important questions remain to be answered, such as whether tDCS effects are specific for the reinforcing effect of nicotine, whether it would also work for other drugs of abuse (eg, alcohol, psychostimulants), and whether it affects the response to natural rewards (eg, sexual activity, appetite). Our preliminary data suggest that the reinforcing effect of food is not altered by transcranial stimulations (no change in food/fluid consumption), but more detailed work is necessary to address this question. A limit in our work is that all of our experiments were carried out in female mice; therefore, additional studies are warranted to determine if our findings can be extrapolated to male mice. A final important consideration is the equivalence of our stimulation paradigm in animal $v s$ humans. Indeed, our stimulation protocol (time, length, repetition) is the same as the one used in clinical trials, but the intensity is lower: $0.2 \mathrm{~mA} v s$ $2 \mathrm{~mA}$. However, in our animal model, the current density is much more elevated due to the small size of the electrode (57.1 vs $0.57 \mathrm{~A} / \mathrm{m}^{2}$ for clinical trials). This is of importance because the area stimulated by the current might be significantly different in mice and in humans, in particular if the size of the brain is taken into account. Future studies will need to determine the minimal amount of current necessary in our model to maintain the efficiency in alleviating abnormal behaviors associated with chronic nicotine consumption, to explore the importance of the polarity, and to assess whether the effects are specific to the area stimulated or simply due to a generalized stimulation of the mouse brain.

Taken together, our findings indicate that (1) our tDCS protocol results in behavioral outcomes similar to the ones observed in clinical trials, (2) exposure to nicotine during adolescence promotes abnormal behaviors during adulthood (depressive-like behavior, increase in the rewarding effect of nicotine), and (3) that this detrimental effect might be prevented by repeated anodal tDCS treatment. Our results also highlight the time dependence of the tDCS effects, which seems to be more prominent 3 weeks after the last tDCS session. On the basis of the research presented here, our experimental animal model provides a framework to investigate the effects of tDCS on smoking craving and, more importantly, to explore the neurobiological changes that underlie the beneficial effects of tDCS on this and other addiction-related behaviors.

\section{FUNDING AND DISCLOSURE}

This work was supported in part by the 'Institut de Recherche Scientifique sur les Boissons' IREB (VVW), the University of Franche-Comté (VVW), and the city of Besançon (SP, VVW).

\section{ACKNOWLEDGEMENTS}

We thank Patrice Andrieu for excellent technical assistance and for his illustration of the tDCS device (Figure 1), MarieAnge Bolard for animal care, and Adriana Caballero for proofreading and editing the manuscript. We are grateful to Jean-Pierre Darnis (Dixi Medical, Besançon, France) for the help in the development of the electrodes for tDCS in mice and to Heinz Steiner for helpful advice and comments.

\section{REFERENCES}

Bindman LJ, Lippold OC, Redfearn JW (1964). The action of brief polarizing currents on the cerebral cortex of the rat (1) during current flow and (2) in the production of long-lasting aftereffects. J Physiol 172: 369-382.

Bindman LJ, Richardson HC (1969). Persisting changes in the firing pattern of single cortical units responding at short latency to weak somatic stimuli in the anaesthetized rat. J Physiol 202: 53P-55P.

Boggio PS, Ferrucci R, Rigonatti SP, Covre P, Nitsche M, PascualLeone A et al (2006). Effects of transcranial direct current stimulation on working memory in patients with Parkinson's disease. J Neurol Sci 249: 31-38.

Boggio PS, Liguori P, Sultani N, Rezende L, Fecteau S, Fregni F (2009). Cumulative priming effects of cortical stimulation on smoking cue-induced craving. Neurosci Lett 463: 82-86.

Brody AL, Mandelkern MA, London ED, Childress AR, Lee GS, Bota RG et al (2002). Brain metabolic changes during cigarette craving. Arch Gen Psychiatry 59: 1162-1172.

Brunoni AR, Ferrucci R, Fregni F, Boggio PS, Priori A (2012). Transcranial direct current stimulation for the treatment of major depressive disorder: a summary of preclinical, clinical and translational findings. Prog Neuropsychopharmacol Biol Psychiatry 39: 9-16.

Due DL, Huettel SA, Hall WG, Rubin DC (2002). Activation in mesolimbic and visuospatial neural circuits elicited by smoking cues: evidence from functional magnetic resonance imaging. $\mathrm{Am}$ J Psychiatry 159: 954-960.

Feil J, Zangen A (2010). Brain stimulation in the study and treatment of addiction. Neurosci Biobehav Rev 34: 559-574.

Ferrucci R, Bortolomasi M, Vergari M, Tadini L, Salvoro B, Giacopuzzi M et al (2009). Transcranial direct current stimulation in severe, drug-resistant major depression. J Affect Disord 118: 215-219.

Fraser PE, Rosen AC (2012). Transcranial direct current stimulation and behavioral models of smoking addiction. Front Psychiatry 3: 79.

Fregni F, Boggio PS, Nitsche M, Bermpohl F, Antal A, Feredoes E et al (2005). Anodal transcranial direct current stimulation of prefrontal cortex enhances working memory. Exp Brain Res 166: 23-30.

Fregni F, Liguori P, Fecteau S, Nitsche MA, Pascual-Leone A, Boggio PS (2008). Cortical stimulation of the prefrontal cortex with transcranial direct current stimulation reduces cueprovoked smoking craving: a randomized, sham-controlled study. J Clin Psychiatry 69: 32-40.

Hughes JR, Stead LF, Lancaster T (2007). Antidepressants for smoking cessation. Cochrane Database Syst Rev CD000031. doi:10.1002/14651858.CD000031.pub3.

Iniguez SD, Warren BL, Parise EM, Alcantara LF, Schuh B, Maffeo ML et al (2009). Nicotine exposure during adolescence induces a depression-like state in adulthood. Neuropsychopharmacology 34: 1609-1624.

Karreman M, Moghaddam B (1996). The prefrontal cortex regulates the basal release of dopamine in the limbic striatum: an effect mediated by ventral tegmental area. J Neurochem 66: 589-598. 
Lam RW (2012). Onset, time course and trajectories of improvement with antidepressants. Eur Neuropsychopharmacol 22(Suppl 3): S492-S498.

Liebetanz D, Fregni F, Monte-Silva KK, Oliveira MB, Amancio-dosSantos A, Nitsche MA et al (2006). After-effects of transcranial direct current stimulation (tDCS) on cortical spreading depression. Neurosci Lett 398: 85-90.

Liebetanz D, Koch R, Mayenfels S, Konig F, Paulus W, Nitsche MA (2009). Safety limits of cathodal transcranial direct current stimulation in rats. Clin Neurophysiol 120: 1161-1167.

McBride D, Barrett SP, Kelly JT, Aw A, Dagher A (2006). Effects of expectancy and abstinence on the neural response to smoking cues in cigarette smokers: an fMRI study. Neuropsychopharmacology 31: 2728-2738.

Nitsche MA, Boggio PS, Fregni F, Pascual-Leone A (2009). Treatment of depression with transcranial direct current stimulation (tDCS): a review. Exp Neurol 219: 14-19.

Nitsche MA, Nitsche MS, Klein CC, Tergau F, Rothwell JC, Paulus W (2003). Level of action of cathodal DC polarisation induced inhibition of the human motor cortex. Clin Neurophysiol 114: 600-604.

Nitsche MA, Paulus W (2000). Excitability changes induced in the human motor cortex by weak transcranial direct current stimulation. J Physiol 527(Pt 3): 633-639.

O'Brien CP (2008). Review. Evidence-based treatments of addiction. Philos Trans R Soc Lond B Biol Sci 363: 3277-3286.

Ohn SH, Park CI, Yoo WK, Ko MH, Choi KP, Kim GM et al (2008). Time-dependent effect of transcranial direct current stimulation on the enhancement of working memory. Neuroreport 19: 43-47.

Paxinos G, Franklin K (2001). The mouse brain in stereotaxic coordinate, 2nd edn. Academic Press.
Porsolt RD, Le Pichon M, Jalfre M (1977). Depression: a new animal model sensitive to antidepressant treatments. Nature 266: 730-732.

Purpura DP, McMurtry JG (1965). Intracellular Activities and evoked potential changes during polarization of motor cortex. J Neurophysiol 28: 166-185.

Rigonatti SP, Boggio PS, Myczkowski ML, Otta E, Fiquer JT, Ribeiro RB et al (2008). Transcranial direct stimulation and fluoxetine for the treatment of depression. Eur Psychiatry 23: $74-76$.

Stagg CJ, Nitsche MA (2011). Physiological basis of transcranial direct current stimulation. Neuroscientist 17: 37-53.

Stead LF, Lancaster T (2012). Combined pharmacotherapy and behavioural interventions for smoking cessation. Cochrane Database Syst Rev 10: CD008286.

Taber MT, Fibiger HC (1993). Electrical stimulation of the medial prefrontal cortex increases dopamine release in the striatum. Neuropsychopharmacology 9: 271-275.

Taber MT, Fibiger HC (1995). Electrical stimulation of the prefrontal cortex increases dopamine release in the nucleus accumbens of the rat: modulation by metabotropic glutamate receptors. J Neurosci 15(5 Pt 2): 3896-3904.

Walf AA, Frye CA (2007). The use of the elevated plus maze as an assay of anxiety-related behavior in rodents. Nature Protocols 2: 322-328.

Wilson SJ, Sayette MA, Fiez JA (2004). Prefrontal responses to drug cues: a neurocognitive analysis. Nat Neurosci 7: 211-214.

Zaehle T, Sandmann P, Thorne JD, Jancke L, Herrmann CS (2011). Transcranial direct current stimulation of the prefrontal cortex modulates working memory performance: combined behavioural and electrophysiological evidence. BMC Neurosci 12: 2. 